\title{
Efficient QCA Exclusive-or and Multiplexer Circuits Based on a Nanoelectronic-Compatible Designing Approach
}

\author{
Amir Mokhtar Chabi, ${ }^{1}$ Samira Sayedsalehi, ${ }^{2,3}$ Shaahin Angizi, ${ }^{3}$ and Keivan Navi ${ }^{3}$ \\ ${ }^{1}$ Department of Computer, Faculty of Engineering, Persian Gulf University, Bushehr 7516913817, Iran \\ ${ }^{2}$ Faculty of Computer Engineering, Islamic Azad University, South Tehran Branch, Tehran 443511365, Iran \\ ${ }^{3}$ School of Computer Science, Institute for Research in Fundamental Sciences (IPM), Tehran 1953833511, Iran \\ Correspondence should be addressed to Samira Sayedsalehi; s.sayedsalehi@srbiau.ac.ir
}

Received 17 April 2014; Revised 13 June 2014; Accepted 28 June 2014; Published 16 October 2014

Academic Editor: Antonios Gasteratos

Copyright (C) 2014 Amir Mokhtar Chabi et al. This is an open access article distributed under the Creative Commons Attribution License, which permits unrestricted use, distribution, and reproduction in any medium, provided the original work is properly cited.

\begin{abstract}
Quantum-dot cellular automata (QCA) are a transistorless computation approach which encodes binary information via configuration of charges among quantum dots. The fundamental QCA logic primitives are majority and inverter gates which can be utilized to design various QCA circuits. This study presents a novel approach to designing efficient QCA-based circuits based on Boolean expressions achieved from reconfiguration of five-input and three-input majority gates. Whereas the multiplexer and Exclusive-or are the most important fundamental logical circuits in digital systems, designing efficient and single layer structures without coplanar cross-over wiring is advantageous in QCA technology. In order to demonstrate the efficiency and usefulness of the proposed approach, simple and dense multiplexer and Exclusive-or structures are implemented. The proposed designs have significant improvement in terms of area, complexity, latency, and gate count in comparison to previous designs. The correct logical functionalities of presented structures have been authenticated using QCA designer tool.
\end{abstract}

\section{Introduction}

Due to current serious exiting challenges in conventional transistor technology, researchers are searching to find an alternative to this technology. Among these new technologies, quantum-dot cellular automata (QCA) are a suitable alternative that offers unique features such as small feature size and ultralow power consumption and can operate at $\mathrm{THz}$ frequencies and room temperature $[1,2]$.

The basic elements in QCA are cells; each cell is composed of two mobile electrons that are located in opposite corners according to columbic energy, resulting in two possible polarizations $(P=+1, P=-1)$ as shown in Figure 1(a) [3].

Up to this time, many methods for fabrication of QCA basic cells are suggested such as metal island [4], magnetic [5], semiconductor [6], and molecular QCA [7]. As is discussed in [8], metal dot implementations have proven to be the most successful material systems which are based on single-electron transistors' fabrication techniques. The magnetic implementation is firstly proposed by Cowburn's group and extended by the Porod group and the Bokar group. In the semiconductor physical implementation, the Cavendish group of Smith et al. proved QCA operation in GaAs/AlGaAs heterostructures with confining top-gate electrodes and the group of Kern et al. demonstrated a silicon QCA cell by employing an etching technique to form the dots. Furthermore, based on [8], the Fehlner and Lapinte groups have performed successful molecular synthesis in creating molecules that show the essential bistability.

According to the columbic interaction between electrons in neighboring cells, the basic logic gates in QCA circuits (inverter and majority gates) are constructed as shown in Figures 1(b) and 1(c), respectively [9-11]. The logical functions of three-input majority gate and five-input majority gate [9] (Figure 1(d)) are

$$
M(A, B, C)=A B+B C+A C
$$

$M(A, B, C, D, E)=A B C+A B D+A B E+A C D+A C E$

$$
+A D E+B C D+B C E+B D E+C D E .
$$




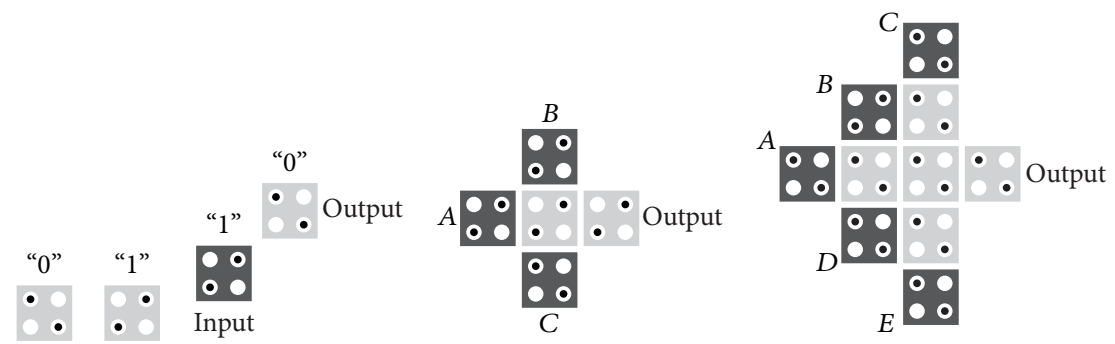

(a)

(b)

(c)

(d)

FIGURE 1: Basic logic cells and gates in QCA: (a) two possible polarizations, (b) inverter, (c) three-input majority gate, and (d) the presented five-input majority gate in [9].

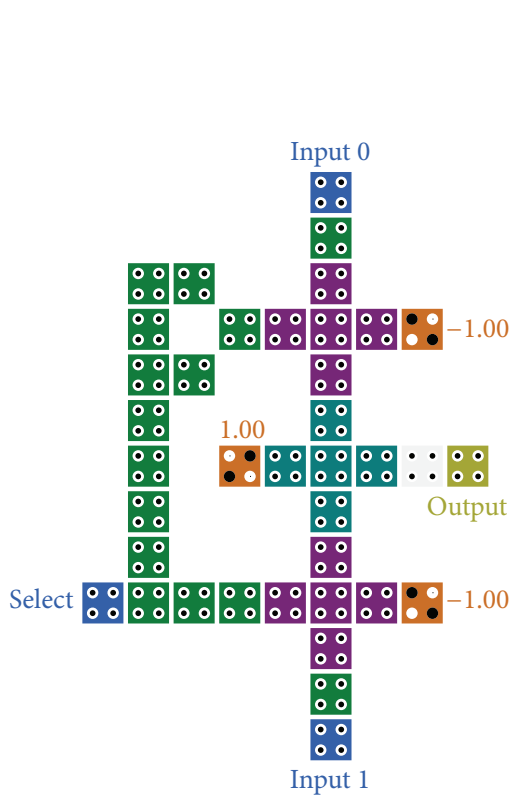

(a)

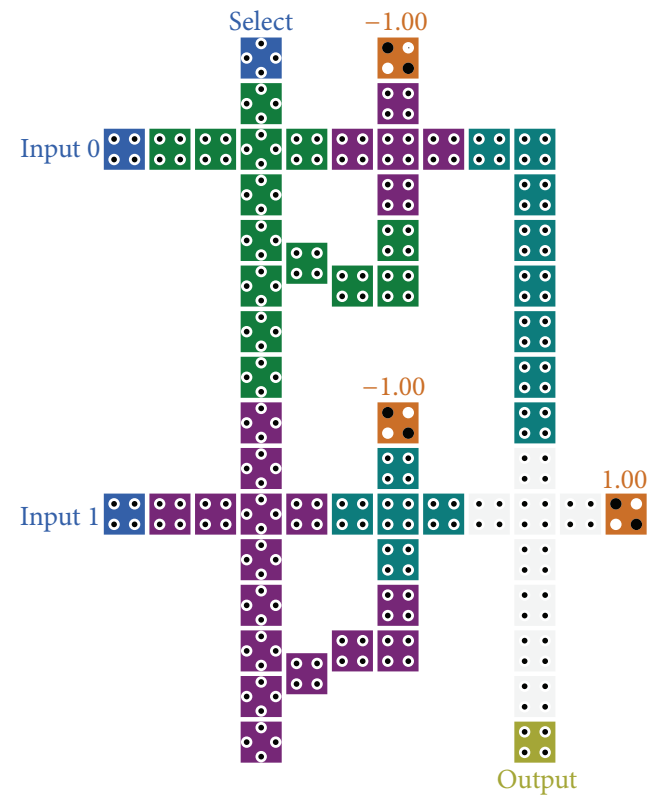

(b)

Figure 2: (a) The presented 2-to-1 multiplexer [17]. (b) The presented 2-to-1 multiplexer [16].

This paper presents a new method to design well-organized QCA circuits that reduces the hardware requirements when compared to previously reported circuits. Multiplexer and Exclusive-or are the most significant components in logical systems, so these circuits are optimized based on this method.

The remainder of this paper is arranged as follows. In Section 2, a review on state-of-the-art designs is provided. Section 3 introduces the new approach to implementing QCA-based structures and proposes efficient and feasible designs for multiplexer and Exclusive-or. In Section 4, we use simulation results obtained from QCADesigner tool to prove the functional correctness of our proposed designs and finally Section 5 concludes the paper.

\section{State-of-the-Art}

As mentioned earlier, the main purpose of this paper is to design two main structures for implementation of various logic circuits, so in this section previous designs are reviewed in order of 2-to-1 multiplexer and Exclusive-or.

2.1. Multiplexer. Multiplexers have a considerable role in the digital systems which allow us to select one of the input's flows for transmitting to the output. Whereas all the logic functions can be built by multiplexers, implementation of multi-input multiplexer in one layer is a remarkable subject. In conventional implementation of multiplexer, there are several structures that have been introduced in [12-22]; all these designs have tried to present improved structure rather than the other. These designs have been implemented using three three-input majority gates in different ways with different propagation delay and consumed cells according to the form of majority gate's concatenation. One of the best proposed structures in terms of complexity and latency is introduced in [17], as shown in Figure 2(a). An innovative methodology for designing 2-to-1 multiplexer is introduced in [16]. This design has a modular structure that consists of 


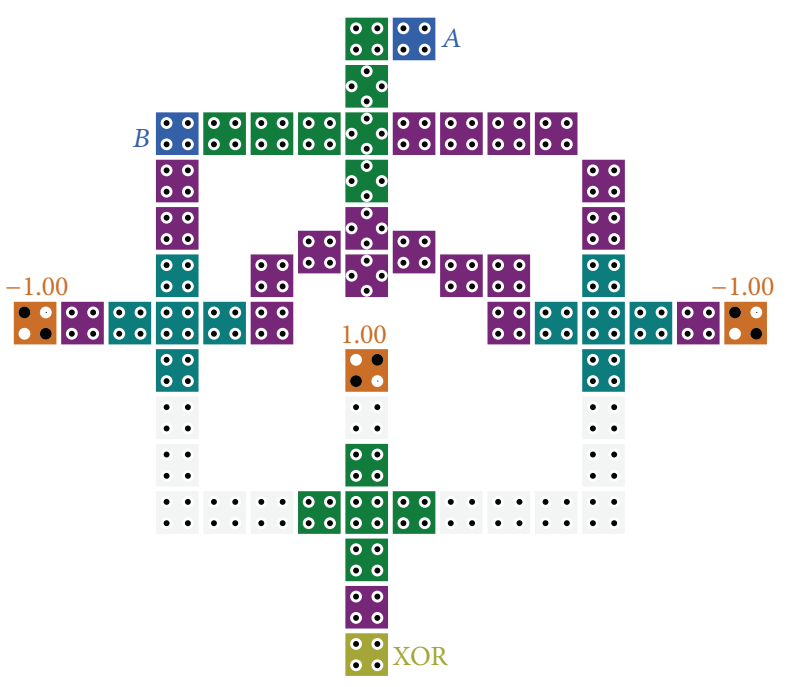

(a)

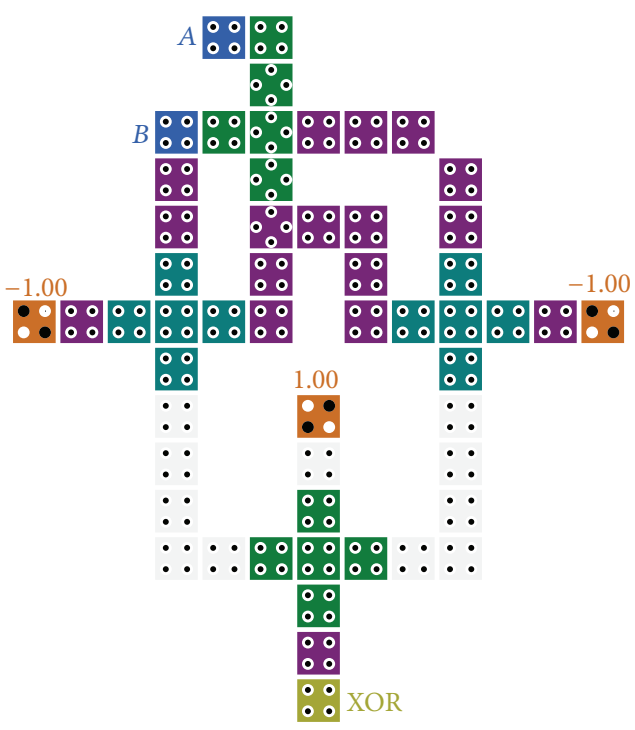

(b)

FIgURE 3: (a) The presented 2-input XOR gate in [23]. (b) The presented 2-input XOR gate using new cell arrangement in [24].

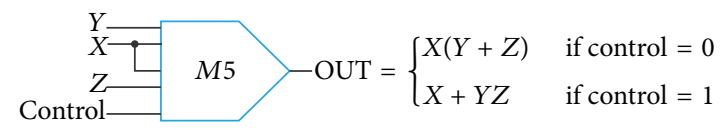

FIgURE 4: Achieved Boolean expressions based on the proposed method.

several elementary blocks as illustrated in Figure 2(b). It is noteworthy that the latency of the circuits in large scales has been diminished by utilizing the presented methodology.

These designs have been implemented according to the following equation:

$$
\begin{aligned}
\text { Output }=\text { Maj }(\text { Maj }(\text { Input } 0, \overline{\text { Select }}, 0), \\
\text { Maj }(\text { Input } 1, \text { select, } 0), 1) .
\end{aligned}
$$

2.2. Exclusive-or. Due to the momentous usage of Exclusiveor component in various tasks such as parity checking and detection and correction mechanism in the receiver and sender units, designing an efficient and high speed Exclusive-or is one of the most important challenges in QCA studies. According to the position of the input signals in the Exclusive-or structure, most of the presented designs are implemented based on the multilayer or coplanar crossover wiring. In $[23,24]$, useful implementations of XOR gate are presented which use coplanar cross-over wiring, as demonstrated in Figure 3. These designs have a similar 1.5 clock cycle delay for transmitting input signals to the output.

\section{Proposed Designing Approach}

The main building block of QCA circuits is majority gate and consequently the other logic circuits are implemented based on majority gate networks. In this section, we are going to propose a novel designing approach to implementing QCA logic circuits with least hardware overhead. To overcome this goal, in addition to three-input majority gate, we have employed the five-input majority gate. In this approach, some functional logic circuits are implemented by configuration of five-input majority gate inputs.

As shown in Figure 4, $X, Y$, and $Z$ are labeled as the main inputs and the control input is labeled as control line. In addition, one of these inputs has twice the effect of the other inputs on five-input majority gate. By setting control line to "1" logic value, the Boolean function $X+Y Z$ is obtained. Furthermore, logical function $X(Y+Z)$ can be achieved by changing the value of the control line to " 0 ."

By using the five-input majority function (2), we get the following equations:

$$
\begin{gathered}
M 5(Y, X, X, Z, 0)=Y X+Y X Z+Y X Z+X Z=X(Y+Z), \\
M 5(Y, X, X, Z, 1)=Y X+Y X Z+Y X+Y X+Y X+Y Z \\
+X Z+X+X Z+X Z=X+Y Z .
\end{gathered}
$$

It is worth mentioning that these functions need only one five-input majority gate for implementation. However, these functions are designed with two three-input majority gates in the conventional method.

From the achieved equations, we can conclude that most of the combinational and sequential circuits can be 


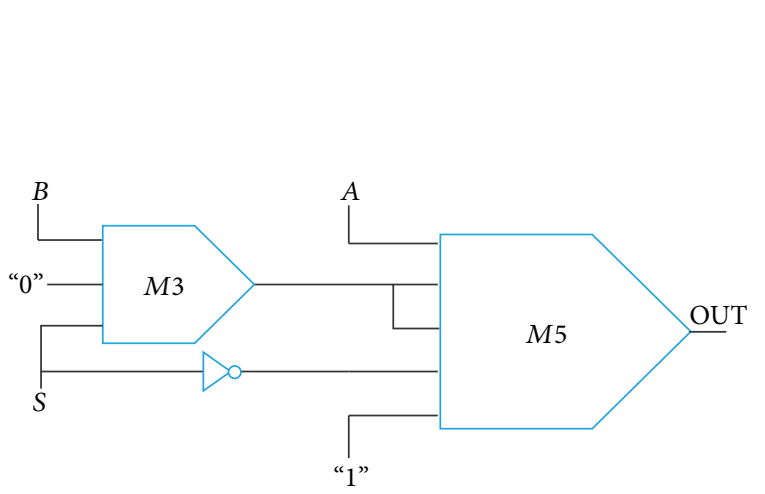

(a)

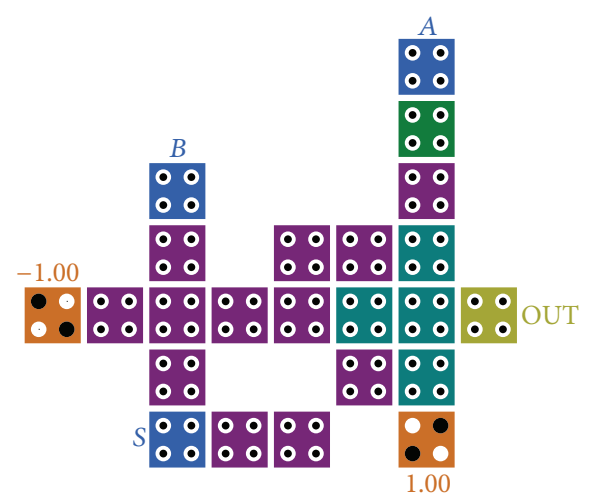

(b)

FIGURE 5: (a) Schematic of the proposed 2-to-1 multiplexer based on new approach. (b) QCA implementation of 2-to-1 multiplexer.

TABLE 1: Truth table of functionality of proposed circuit.

\begin{tabular}{cccccc}
\hline$S$ & $A$ & $B$ & $X=S \cdot B$ & $2 X+Y+Z+1$ & OUT \\
\hline 0 & 0 & 0 & 0 & 2 & 0 \\
0 & 0 & 1 & 0 & 2 & 0 \\
0 & 1 & 0 & 0 & 3 & 1 \\
0 & 1 & 1 & 0 & 3 & 1 \\
1 & 0 & 0 & 0 & 1 & 0 \\
1 & 0 & 1 & 1 & 3 & 1 \\
1 & 1 & 0 & 0 & 2 & 0 \\
1 & 1 & 1 & 1 & 4 & 1 \\
\hline
\end{tabular}

constructed by assigning proper functions or fixed values to their parameters. The next section provides implementation steps of multiplexer and Exclusive-or circuits based on this method.

3.1. Multiplexer Design. As noted above, a feasible design for 2-to-1 multiplexer can be obtained by utilizing the proposed novel method. According to the 2-to-1 multiplexer function, the inputs of logical function $X+Y Z$ should be changed as in Figure 5(a).

The logic function $B \cdot S$ should be fed to input $X$ and also the inputs $A$ and $\bar{S}$ should be fed to the inputs $Y$ and $Z$, respectively. For implementation of logical function $B S$, a three-input majority gate has been used. It is to be noted that only two majority gates and one inverter gate are used for implementing this structure.

By applying this method, the equation of 2-to-1 multiplexer is defined as follows:

$$
\begin{gathered}
M 5(A, M 3(B, 0, S), M 3(B, 0, S), \bar{S}, 1), \\
M 5(A, B S, B S, \bar{S}, 1)=A \bar{S}+B S .
\end{gathered}
$$

To clarify the correct functionality of the proposed design in detail, the truth table of proposed circuit is shown in Table 1 with three output columns. The first column presents eight possible combinations of three input cells $(S, A$, and $B)$. The second column demonstrates the output of three-input majority gate which produces the logical function $B \cdot S$. The summation of five-input majority gate inputs is shown in the third column and the last column illustrates the main output of the proposed 2-to-1 multiplexer circuit.

As it is obvious in Figure 5(b), the latency of proposed multiplexer is 0.75 clock cycle, so this design is the fastest in comparison to previous mentioned designs.

3.2. Exclusive-or Design. In this section, we propose our new high speed and single layer two-input XOR gate using the $X+Y Z$ equation mentioned above. The applied equation which is achieved using the majority gate functions is shown in (9). As illustrated in Figure 6(a), the output of three-input majority gate $(\bar{A} B)$ with twice the effect and inverse of $B$ signal and $A$ signal are assigned to the five-input majority gate in the similar clocking zone. Consequently, the Exclusive-or of $A$ and $B$ signals is produced in the next clocking zone. Considering the similar procedure, we get the equation of two-input XOR gate as follows:

$$
\begin{gathered}
M 5(A, M 3(\bar{A}, 0, B), M 3(\bar{A}, 0, B), \bar{B}, 1), \\
M 5(A, \bar{A} B, \bar{A} B, \bar{B}, 1)=\bar{A} B+A \bar{B} .
\end{gathered}
$$

The propagation delay of the presented two-input XOR gate is 0.75 clock cycle with huge reduction in cell counts and area occupation. The significant contribution of this design is implemented in signal layer without using rotated cells in comparison to previous designs. This structure can be expanded to the larger scale by cascading several two-input XOR gates. As example, the schematic of four-input XOR gate and its QCA implementation are shown in Figure 7.

\section{Simulation Results}

QCADesigner is a well-known simulation tool generally expanded for evaluating QCA logic circuits; this tool has two different simulation engines which are called bistable approximation and coherence vector. The bistable approximation engine calculates state of a single cell using a time-independent approach with kink energy formula that 


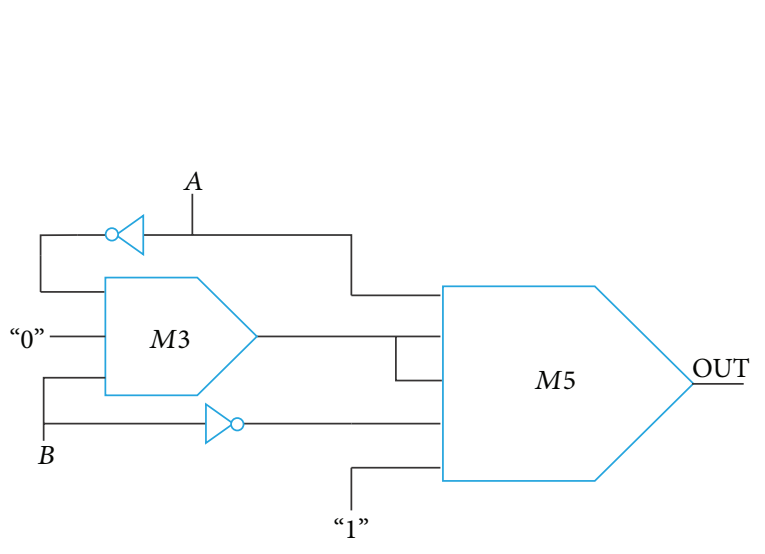

(a)

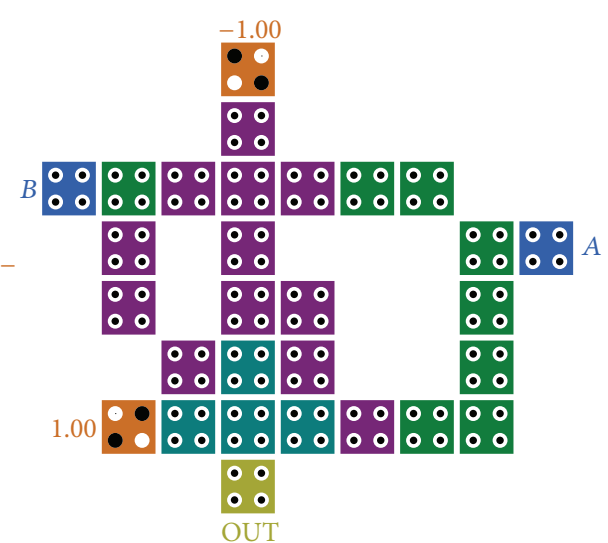

(b)

FIGURE 6: (a) Schematic of the proposed two-input Exclusive-or based on new approach. (b) QCA implementation of two-input Exclusive-or.

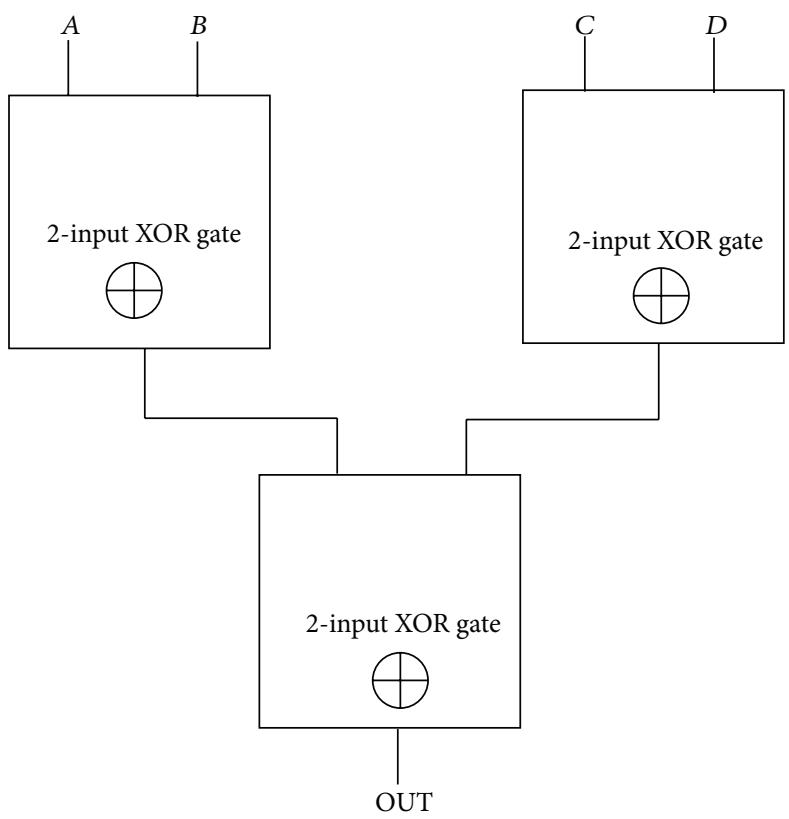

(a)

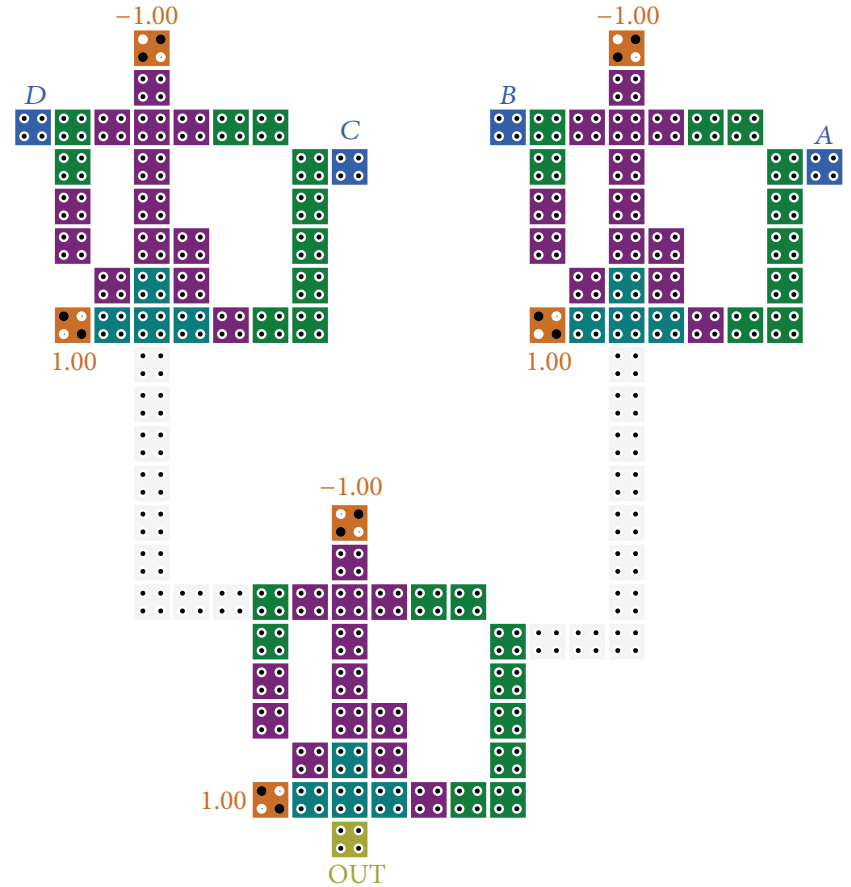

(b)

FIGURE 7: (a) Block diagram of four-input XOR gate. (b) QCA layout of four-input XOR gate based on cascading of the proposed XOR gate in Figure 6(b).

calculates cost of two cells having opposite polarizations, so simulation time in this engine is reduced. The coherence vector model considers the time-dependent state of a cell in interaction with the other cells through the same kink energy formula [25-27].

In QCADesigner software, each single cell (standard, rotated) can act in four modes (input, output, fixed, and normal) which is shown in Figure 8.

In this section, correct functionality of the proposed structures is authenticated using QCADesigner tool version

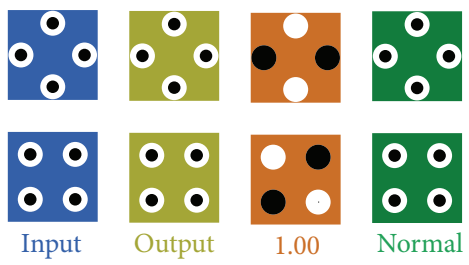
(a)
(b)
(c)
(d)

FIGURE 8: QCADesigner cells: (a) input cell, (b) output cell, (c) fixed polarization cell, and (d) normal cell. 


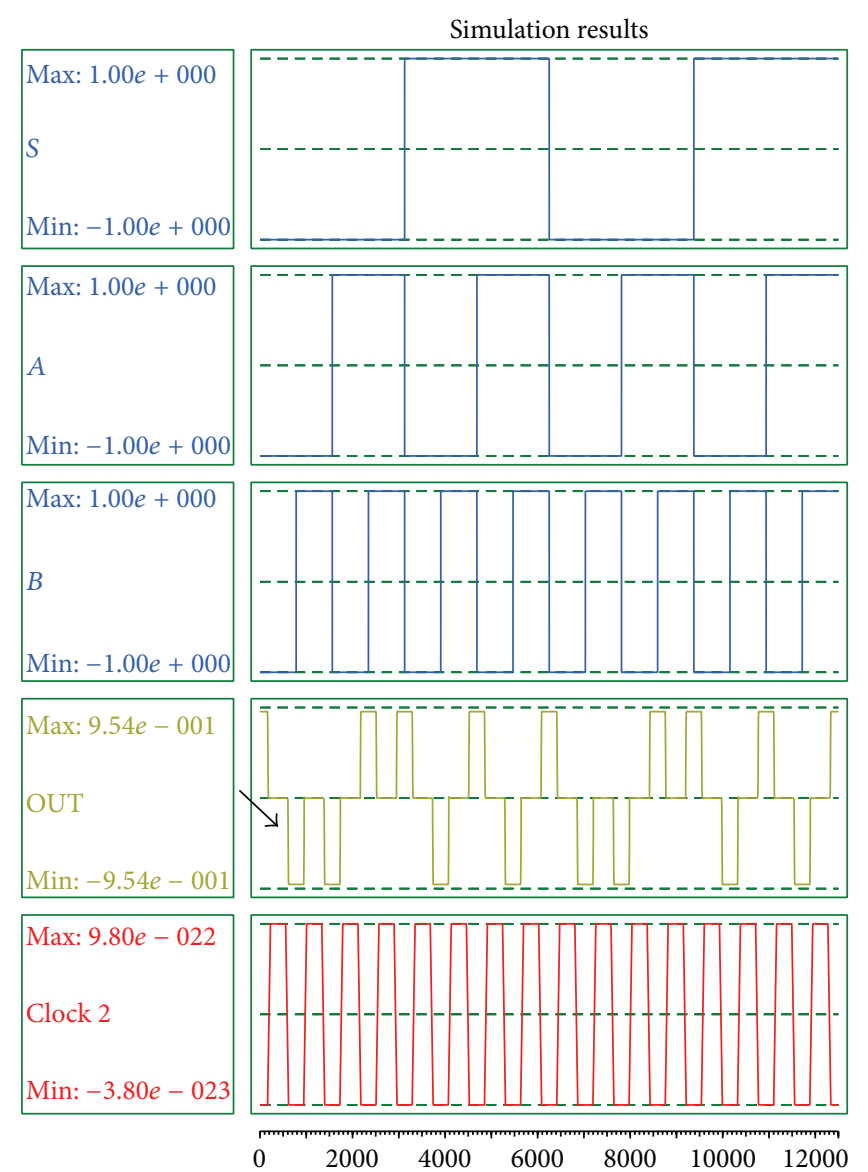

(a)

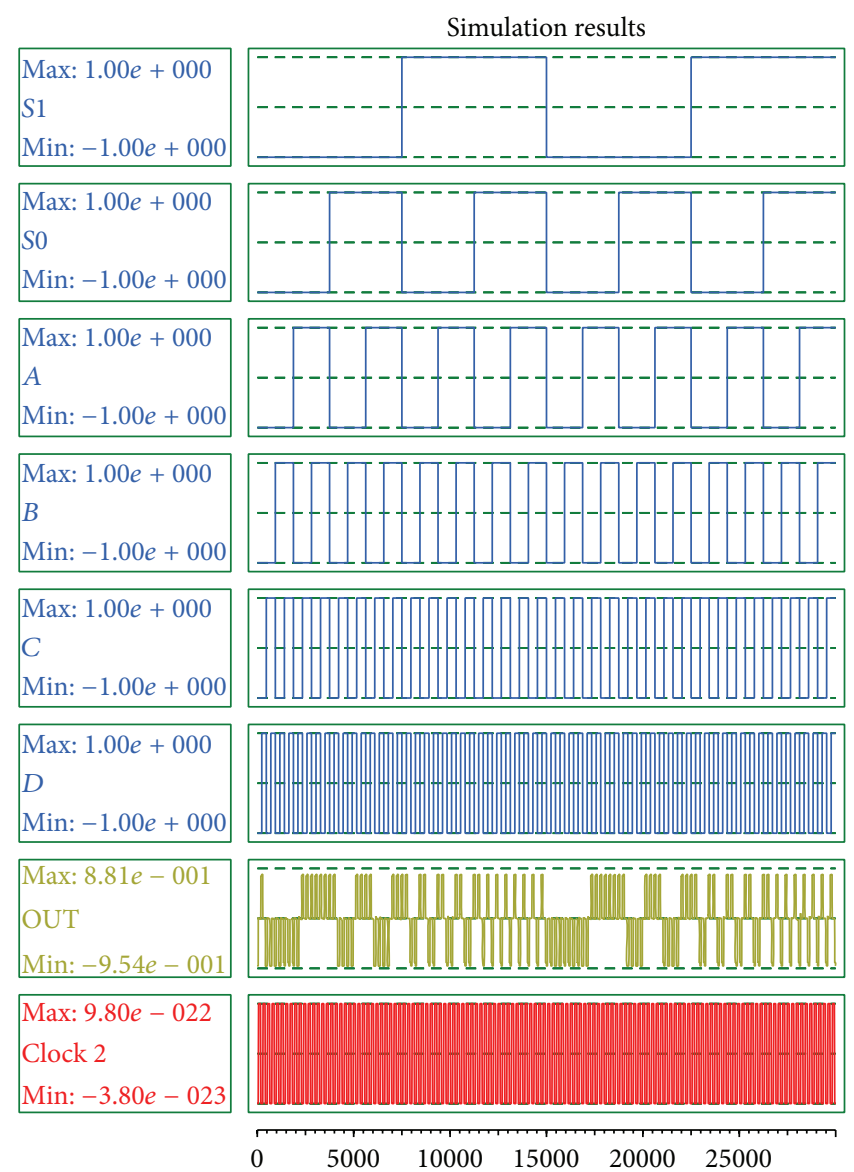

(b)

Figure 9: (a) Simulation result of the proposed 2-to-1 multiplexer. (b) Simulation result of 4-to-1 multiplexer.

TABLE 2: Bistable approximation parameters model.

\begin{tabular}{lc}
\hline Parameter & Value \\
\hline Cell size & $18 \mathrm{~nm}$ \\
Number of samples & 50000 \\
Convergence tolerance & 0.001000 \\
Radius of effect & $65.000000 \mathrm{~nm}$ \\
Relative permittivity & 12.900000 \\
Clock low & $3.800000 e-023 \mathrm{~J}$ \\
Clock high & $9.800000 e-022 \mathrm{~J}$ \\
Clock shift & 0 \\
Clock amplitude factor & 2.000000 \\
Layer separation & 11.500000 \\
Maximum iterations per sample & 100 \\
\hline
\end{tabular}

2.0.3 [25]. Each one of the circuits is examined under both simulation engines (bistable approximation and coherence vector) and similar outcomes are achieved. Tables 2 and 3 illustrate the applied parameters in bistable approximation and coherence vector simulation engines, respectively.

The analysis of output waveforms verifies the accuracy and efficiency of presented designs in comparison to stateof-the-art designs. The simulation results of proposed 2-to-1
TABLE 3: Coherence vector parameters model.

\begin{tabular}{lc}
\hline Parameter & Value \\
\hline Temperature & $1.000000 \mathrm{~K}$ \\
Relaxation time & $4.1356675 e-14 \mathrm{~s}$ \\
Time step & $1.000000 e-016 \mathrm{~s}$ \\
Total simulation time & $7.000000 e-011 \mathrm{~s}$ \\
Clock high & $9.800000 e-022 \mathrm{~J}$ \\
Clock low & $3.800000 e-023 \mathrm{~J}$ \\
Clock shift & $0.000000 e+000$ \\
Clock amplitude factor & 2.000000 \\
Radius of effect & $80.000000 \mathrm{~nm}$ \\
Relative permittivity & 12.900000 \\
Layer separation & $11.500000 \mathrm{~nm}$ \\
\hline
\end{tabular}

multiplexer and 4-to-1 multiplexer are illustrated in Figures 9(a) and 9(b), respectively. As is clear in Figure 9(a), the first meaningful output appears in 0.75 clock cycle.

In Figures 10(a) and 10(b), simulation results of twoinput and four-input Exclusive-or circuits are authenticated. Latency of the two-input and four-input XOR gates is 0.75 and 2.75 clock cycle, respectively. 


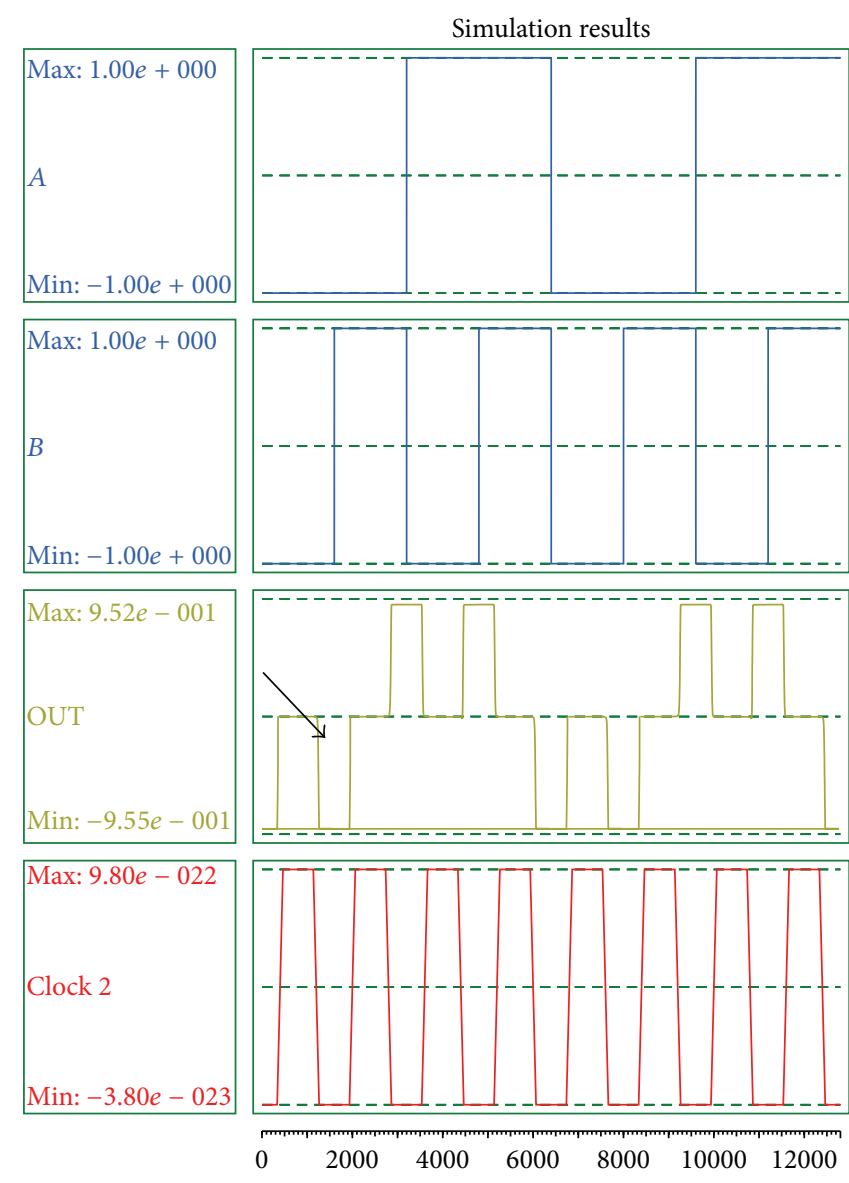

(a)

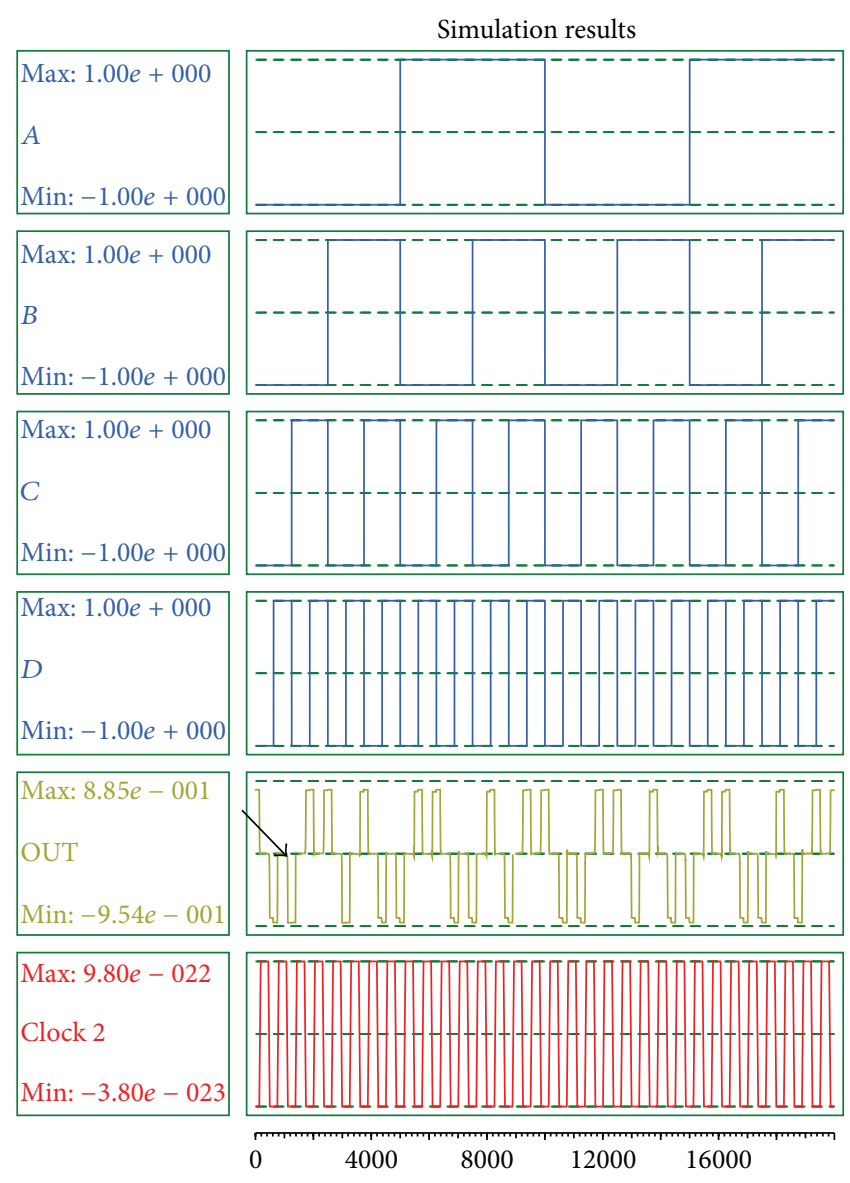

(b)

FIGURE 10: (a) Simulation result of the proposed two-input XOR gate. (b) Simulation result of four-input XOR gate.

TABLE 4: Comparison results of the presented multiplexers.

\begin{tabular}{|c|c|c|c|c|c|c|}
\hline & Circuit & $\begin{array}{c}\text { Gate count } \\
\text { (majority gate }+ \text { inverter) }\end{array}$ & Area $\left(\mu \mathrm{m}^{2}\right)$ & Cell count & $\begin{array}{l}\text { Latency } \\
\text { (clock) }\end{array}$ & Cross-over type \\
\hline \multirow{8}{*}{$2: 1$ multiplexer } & {$[12]$} & 4 & 0.08 & 46 & 1 & Multilayer \\
\hline & {$[13]$} & 4 & 0.06 & 36 & 1 & Multilayer \\
\hline & {$[14]$} & 3 & 0.07 & 54 & 1 & Coplanar \\
\hline & {$[15]$} & 3 & 0.09 & 60 & 1 & Coplanar (rotated cells) \\
\hline & {$[16]$} & 3 & 0.07 & 56 & 1 & Coplanar \\
\hline & [17] & 4 & 0.05 & 38 & 1 & Not required \\
\hline & {$[18]$} & 5 & 0.02 & 23 & $3 / 4$ & Not required \\
\hline & The proposed & 3 & 0.03 & 23 & $3 / 4$ & Not required \\
\hline \multirow{8}{*}{$4: 1$ multiplexer } & [19] & 15 & 0.25 & 246 & $1+1 / 4$ & Multilayer \\
\hline & {$[20]$} & 18 & 0.25 & 124 & 2 & Not Required \\
\hline & {$[21]$} & 11 & 0.15 & 154 & 1 & Multilayer \\
\hline & {$[14]$} & 9 & 0.24 & 159 & $2+1 / 4$ & Coplanar \\
\hline & {$[15]$} & 9 & 0.72 & 456 & $2+3 / 4$ & Coplanar \\
\hline & [16] & 11 & 0.25 & 215 & $1+1 / 2$ & Coplanar \\
\hline & {$[22]$} & 14 & 0.08 & 73 & 1 & Coplanar \\
\hline & The proposed & 9 & 0.11 & 82 & $1+3 / 4$ & Not required \\
\hline
\end{tabular}


TABLE 5: The proposed multiplexer improvements in comparison to other previous designs.

\begin{tabular}{|c|c|c|c|c|c|}
\hline Improvement & Circuit & $\begin{array}{c}\text { Gate count } \\
\text { (majority gate }+ \text { inverter) }\end{array}$ & Area & Cell count & Latency \\
\hline \multirow{7}{*}{ 2:1 multiplexer } & {$[12]$} & $25 \%$ & $62 \%$ & $50 \%$ & $25 \%$ \\
\hline & {$[13]$} & $25 \%$ & $50 \%$ & $36 \%$ & $25 \%$ \\
\hline & {$[14]$} & $0 \%$ & $57 \%$ & $57 \%$ & $25 \%$ \\
\hline & {$[15]$} & $0 \%$ & $66 \%$ & $61 \%$ & $25 \%$ \\
\hline & [16] & $0 \%$ & $57 \%$ & $59 \%$ & $25 \%$ \\
\hline & [17] & $25 \%$ & $40 \%$ & $39 \%$ & $25 \%$ \\
\hline & [18] & $40 \%$ & $-50 \%$ & $0 \%$ & $0 \%$ \\
\hline \multirow{7}{*}{$4: 1$ multiplexer } & [19] & $40 \%$ & $56 \%$ & $66 \%$ & $-28 \%$ \\
\hline & [20] & $50 \%$ & $56 \%$ & $33 \%$ & $12 \%$ \\
\hline & {$[21]$} & $18 \%$ & $26 \%$ & $46 \%$ & $-42 \%$ \\
\hline & {$[14]$} & $0 \%$ & $54 \%$ & $48 \%$ & $22 \%$ \\
\hline & {$[15]$} & $0 \%$ & $84 \%$ & $82 \%$ & $36 \%$ \\
\hline & [16] & $18 \%$ & $56 \%$ & $61 \%$ & $-16 \%$ \\
\hline & [22] & $35 \%$ & $-37 \%$ & $-12 \%$ & $-75 \%$ \\
\hline
\end{tabular}

TABLE 6: Comparison results of presented Exclusive-or designs.

\begin{tabular}{lccccc}
\hline Circuit & Gate count & Area $\left(\mu \mathrm{m}^{2}\right)$ & Cell count & $\begin{array}{c}\text { Latency } \\
\text { (Clock) }\end{array}$ & Cross-over type \\
\hline XOR 2 [23] & 5 & 0.09 & 60 & 1.5 & Coplanar (rotated cells) \\
XOR 2 [24] & 5 & 0.08 & 54 & 1.5 & Coplanar (rotated cells) \\
The proposed XOR 2 & 4 & 0.03 & 29 & 0.75 & Not required \\
The proposed XOR 4 & 12 & 0.19 & 106 & 1.75 & Not required \\
The proposed XOR 8 & 28 & 0.60 & 269 & 2.75 & Not required \\
\hline
\end{tabular}

Table 4 comprises the previous works in 2-to-1 and 4-to-1 multiplexer's designs with the proposed multiplexers in terms of hardware requirement and latency.

According to Table 5, it can be concluded that this proposed design results in significant improvements in gate count, area, cell count, and latency. The comparison results of Exclusive-or circuits are illustrated in Table 6. Based on the obtained results, it can be concluded that employing the proposed approach in QCA circuits leads to a considerable optimization in cell count, occupation area, and propagation delay.

\section{Conclusion}

In this paper, the new approach to implementation of QCAbased circuit was introduced. This method is based on the new configuration of five-input majority gate that led to achieve significant Boolean function such as $X+Y \cdot Z$. It is expected that the novel method presented in this paper will produce efficient QCA-based logical circuits such as multiplexer and Exclusive-or. These proposed circuits surpass previous designs in terms of gate count, area, cell count, and latency. Furthermore, the great advantage of the presented approach is that it leads to implementation of these structures in single layer without any cross-over wiring.

\section{Conflict of Interests}

The authors declare that there is no conflict of interests regarding the publication of this paper.

\section{References}

[1] J. M. Seminario, P. A. Derosa, L. E. Cordova, and B. H. Bozard, "A molecular device operating at terahertz frequencies: theoretical simulations," IEEE Transactions on Nanotechnology, vol. 3, no. 1, pp. 215-218, 2004.

[2] R. P. Cowburn and M. E. Welland, "Room temperature magnetic quantum cellular automata," Science, vol. 287, no. 5457, pp. 1466-1468, 2000.

[3] C. S. Lent, P. D. Tougaw, W. Porod, and G. H. Bernstein, "Quantum cellular automata," Nanotechnology, vol. 4, no. 1, pp. 49-57, 1993.

[4] G. Tóth and C. S. Lent, "Quasiadiabatic switching for metalisland quantum-dot cellular automata," Journal of Applied Physics, vol. 85, no. 5, pp. 2977-2984, 1999.

[5] G. H. Bernstein, A. Imre, V. Metlushko et al., "Magnetic QCA systems," Microelectronics Journal, vol. 36, no. 7, pp. 619-624, 2005.

[6] G. L. Snider, A. O. Orlov, I. Amlani et al., "Experimental demonstration of quantum-dot cellular automata," Semiconductor Science and Technology, vol. 13, no. 8, pp. A130-A134, 1998. 
[7] P. D. Tougaw, Quantum cellular automata: computing with quantum dot molecules [Ph.D. thesis], University of Notre Dame, 1996.

[8] N. G. Anderson and S. Bhanja, Field-Coupled Nanocomputing Paradigms, Progress, and Perspectives, Springer, Heidelberg, Germany.

[9] K. Navi, R. Farazkish, S. Sayedsalehi, and M. Rahimi Azghadi, "A new quantum-dot cellular automata full-adder," Microelectronics Journal, vol. 41, no. 12, pp. 820-826, 2010.

[10] P. D. Tougaw and C. S. Lent, "Logical devices implemented using quantum cellular automata," Journal of Applied Physics, vol. 75, no. 3, pp. 1818-1825, 1994.

[11] K. Navi, S. Sayedsalehi, R. Farazkish, and M. R. Azghadi, "Fiveinput majority gate, a new device for quantum-dot cellular automata," Journal of Computational and Theoretical Nanoscience, vol. 7, no. 8, pp. 1546-1553, 2010.

[12] K. Kim, K. Wu, and R. Karri, “The robust QCA adder designs using composable QCA building blocks," IEEE Transactions on Computer-Aided Design of Integrated Circuits and Systems, vol. 26, no. 1, pp. 176-183, 2007.

[13] S. Hashemi, M. R. Azghadi, and A. Zakerolhosseini, "A novel QCA multiplexer design," in Proceedings of the International Symposium on Telecommunications (IST '08), pp. 692-696, August 2008.

[14] H. Balijepalli and M. Niamat, "Design of a nanoscale quantumdot cellular automata configurable logic block for FPGAs," in Proceedings of the 2012 IEEE 55th International Midwest Symposium on Circuits and Systems (MWSCAS '12), pp. 622625, August 2012.

[15] M. Kianpour and R. Sabbaghi-Nadooshan, "A conventional design for CLB implementation of a FPGA in Quantum-dot Cellular Automata (QCA)," in Proceedings of the IEEE/ACM International Symposium on Nanoscale Architectures (NANOARCH '12), pp. 36-42, Amsterdam, Netherlands, July 2012.

[16] V. A. Mardiris and I. G. Karafyllidis, "Design and simulation of modular $2^{n}$ to 1 quantum-dot cellular automata (QCA) multiplexers," International Journal of Circuit Theory and Applications, vol. 38, no. 8, pp. 771-785, 2010.

[17] S. Hashemi and K. Navi, "New robust QCA D flip flop and memory structures," Microelectronics Journal, vol. 43, no. 12, pp. 929-940, 2012.

[18] D. Mukhopadhyay and P. Dutta, "Quantum cellular automata based novel unit 2:1 multiplexer," International Journal of Computer Applications, vol. 43, no. 2, pp. 22-25, 2012.

[19] V. C. Teja, S. Polisetti, and S. Kasavajjala, "QCA based multiplexing of 16 arithmetic \& logical subsystems-a paradigm for nano computing," in Proceedings of the 3rd IEEE International Conference on Nano/Micro Engineered and Molecular Systems (NEMS '08), pp. 758-763, Sanya, China, January 2008.

[20] M. Askari, M. Taghizadeh, and K. Fardad, "Digital design using quantum-dot cellular automata (A nanotechnology method)," in Proceedings of the International Conference on Computer and Communication Engineering (ICCCE '08), pp. 952-955, May 2008.

[21] A. M. Chabi, S. Sayedsalehi, and K. Navi, "New modules for quantum-dot cellular automata AND \& OR gates," Canadian Journal on Electrical and Electronics Engineering, vol. 3, no. 5, pp. 200-208, 2012.

[22] A. Sarkar and D. Mukhopadhyay, "Improved quantum dot cellular automata 4:1 multiplexer circuit unit," SOP Transactions on Nano-Technology, vol. 1, no. 1, pp. 37-44, 2014.
[23] M. T. Niemier, Designing digital systems in quantum cellular automata [M.S. thesis], University of Notre Dame, 2004.

[24] S. Hashemi, R. Farazkish, and K. Navi, "New quantum dot cellular automata cell arrangements," Journal of Computational and Theoretical Nanoscience, vol. 10, no. 4, pp. 798-809, 2013.

[25] QCADesigner Documentation, http://www.qcadesigner.ca/.

[26] K. Kim, K. Wu, and R. Karri, "Quantum-dot Cellular Automata design guideline," IEICE Transactions on Fundamentals of Electronics, Communications and Computer Sciences, vol. E89A, no. 6, pp. 1607-1614, 2006.

[27] K. Walus, T. J. Dysart, G. A. Jullien, and R. A. Budiman, "QCADesigner: a rapid design and simulation tool for quantum-dot cellular automata," IEEE Transactions on Nanotechnology, vol. 3, no. 1, pp. 26-31, 2004. 

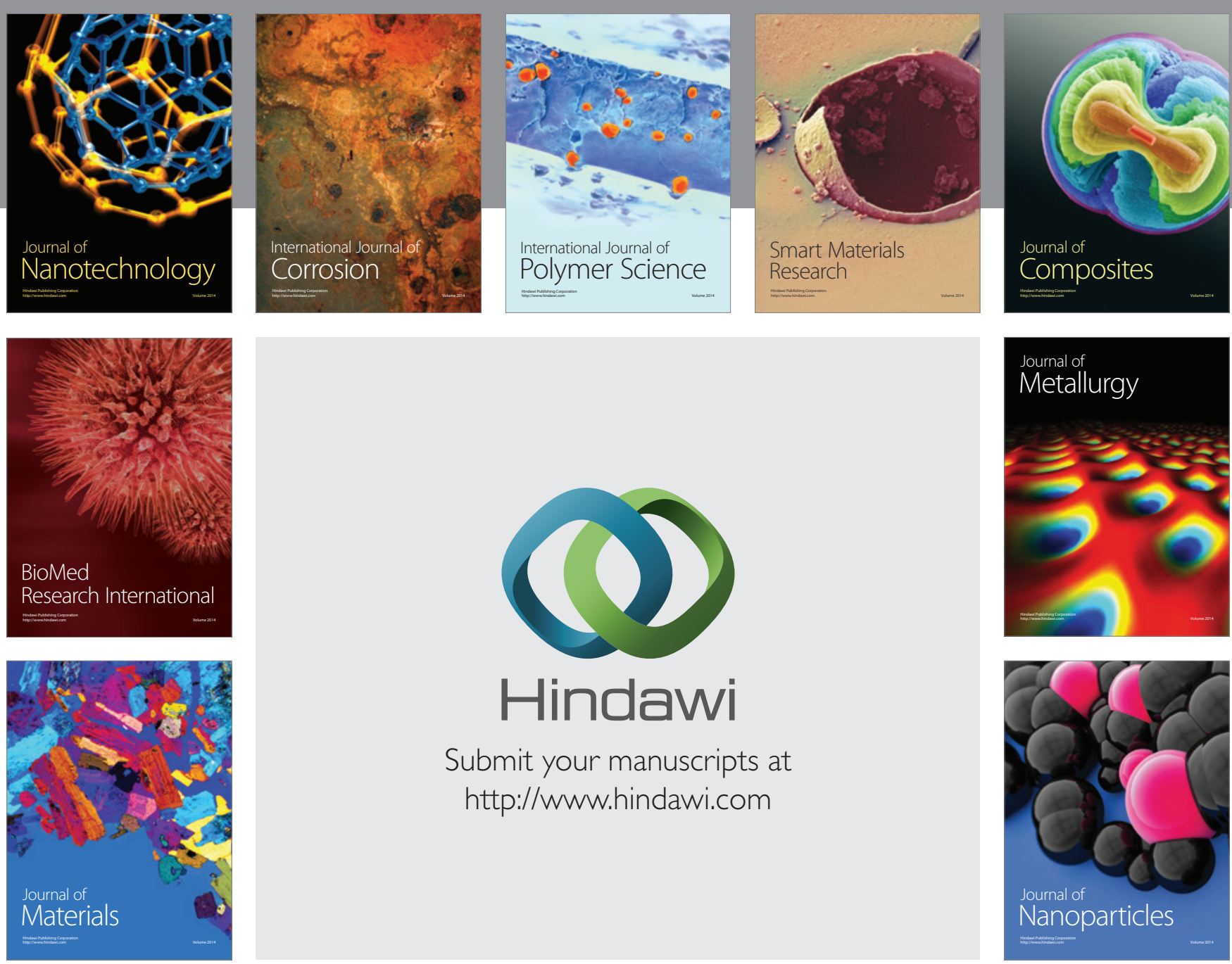

Submit your manuscripts at http://www.hindawi.com
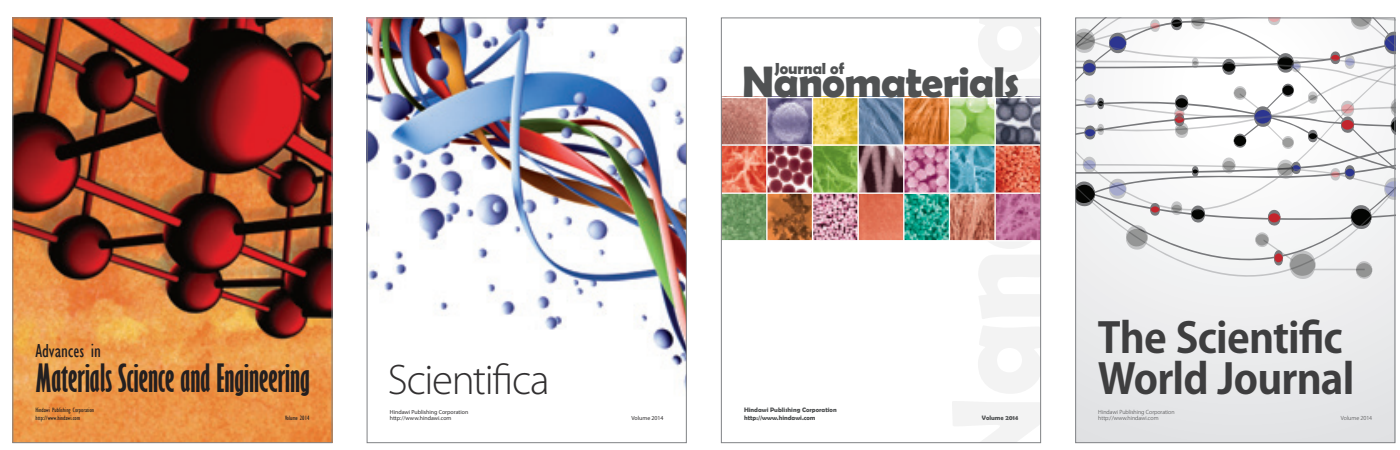

\section{The Scientific World Journal}
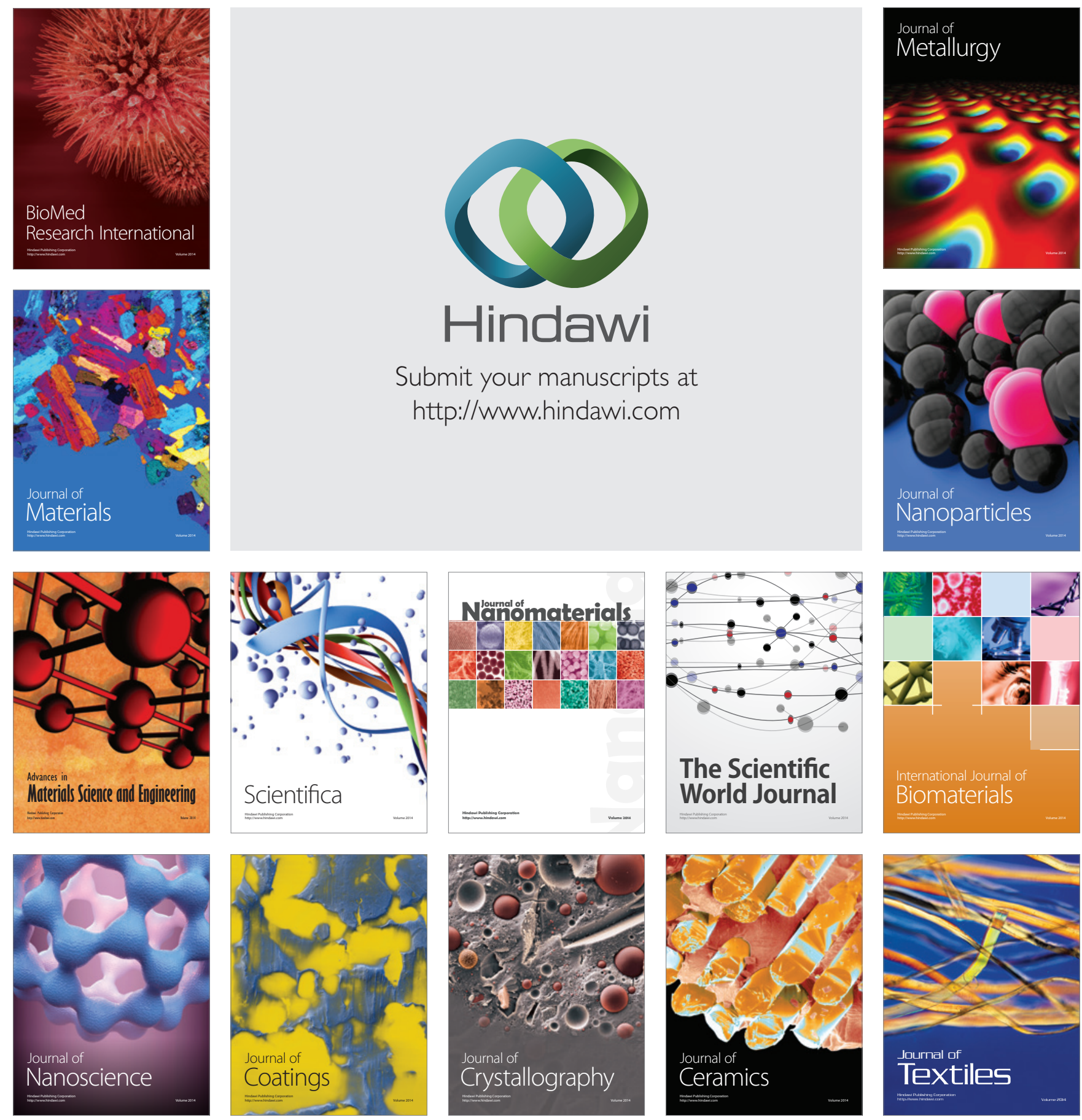